\title{
Análisis fisicoquímico de la cabuya azul
}

Mooner Aurelio Lavado Soto* Roberto Robles CALDERÓN** Julio Antolin Yenque Dedios ***

\section{RESUMEN}

En las zonas rurales de Huancavelica, Huánuco, Ayacucho, Apurímac y Cajamarca, abunda un recurso natural llamado cabuya azul. Se tiene información que en los departamentos del sur se procesa artesanalmente el jugo de cabuya para obtener mie y panela, los cuales se utilizan como edulcorante. Dada esta situación es necesario realizar un análisis fisicoquímico del jugo de la cabuya azul a fin de identificar algunas propiedades, principalmente el contenido de inulina que tiene propiedades dietéticas. Las pruebas experimentales realizadas demuestran que el jugo de este recurso natural contiene alto contenido de inulina y sólidos totales (fructosa, glucosa y otros). En base a lo anterior es factible su transformación industrial, coadyuvando de esta manera a mejorar el nivel de vida de la población rural donde abunda este recurso natural.

Palabras clave: cabuya azul, fructosa, grados brix, inulina, viscosidad

Physicochemical analysis of the blue agave

\section{ABSTRAC}

In rural areas of Huancavelica, Huánuco, Ayacucho, Apurimac and Cajamarca. There is an abundant natural resource called blue sisal. There is information that in the southern departments the sisal juice is processed handmade to obtain honey and brown sugar, which are used as a sweetener. Given this situation, it is needed a blue sisal juice physicochemical analysis to identify some properties, mainly the content of inulin having dietary properties. Experimental tests show that this juice found in natural resource contains high content of inulin and total solids (fructose, glucose and others). Based on the above, the industrial processing is feasible; contributing in this way to improve the standard of living of the rural population where this natural resource is abundant.

Keywords: blue sisal (blue cabuya), degrees brix, fructose, viscosity, inulin

\section{INTRODUCCIÓN}

En algunas regiones del Perú la cabuya azul abunda en forma silvestre, cuyo tronco (piña) de esta planta es rico en fructanos, que consiste de una mezcla compleja, conteniendo principalmente de estructura altamente ramificada, en el cual la inulina constituye una fracción del contenido total de fructanos. Constituyendo este este recurso natural una fuente de inulina, por lo que es importante promover su transformación industrial, coadyuvando a mejorar el nivel de vida de la población rural donde abunda este recurso.

\section{PLANTEAMIENTO DEL ESTUDIO (2)}

La cabuya azul abunda en algunas zonas rurales de Huancavelica, Huánuco, Ayacucho, Apurímac y Cajamarca. Dadas las bondades alimenticias y medicinales de la inulina, el presente estudio de investigación está orientado a la obtención del jugo o agua miel de cabuya, cuyo análisis fisicoquímico debe demostrar un contenido apreciable de inulina.

\section{INFORMACIÓN GENERAL SOBRE EL RECURSO NATURAL}

\section{Clasificación botánica}

La cabuya azul pertenece a la familia Agavácea, género Agave y especie Agave Americana.

\section{Marco teórico}

La mayor parte de la piña de agave está compuesta por inulina, un carbohidrato ramificado que actúa como fuente de reserva de energía en la planta. La inulina se puede extraer fácilmente e hidrolizar para dar oligofructosa, fructosa, sacarosa y glucosa. Tanto la inulina como la oligofructosa presentan características dietéticas que hacen interesante su obtención y aplicación en el campo de la tecnología de alimentos.

Las inulinas son polímeros ramificados de moléculas de fructosa unidas por enlaces glucosídicos y contienen una molécula de glucosa en el extremo reductor de la cadena. Se encuentran en algunas plantas monocotiledóneas, entre ellas las familias

\footnotetext{
* Ing. Quimico, Docente Principal, FII - UNMSM. E-mail: mlavados@unmsm.edu.pe

** Ing. Quimico, Docente Auxiliar, FQIQ - UNMSM. E-mail: rroblesc1@unmsm.edu.pe.lderón

*** Magíster en Ing. Industrial, Docente Asociado, FII - UNMSM. E-mail: Jyenque@yahoo.es
} 
Agavácea. El grado de polimerización llega a ser de 200 unidades de fructosa, aunque generalmente su tamaño oscila entre 30 y 60 unidades de fructuosa. La fórmula de la inulina es:

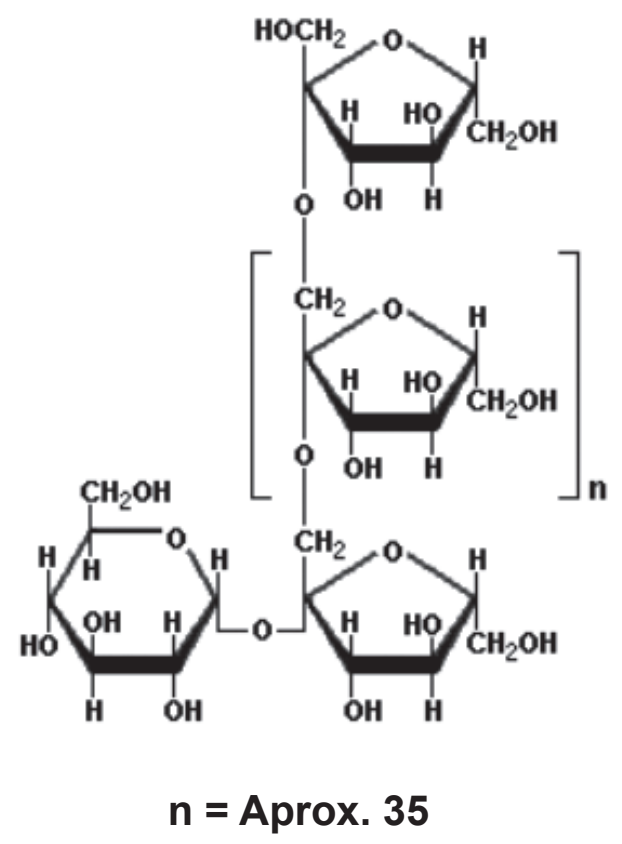

Figura 1. Cabuya azul de Huancavelica

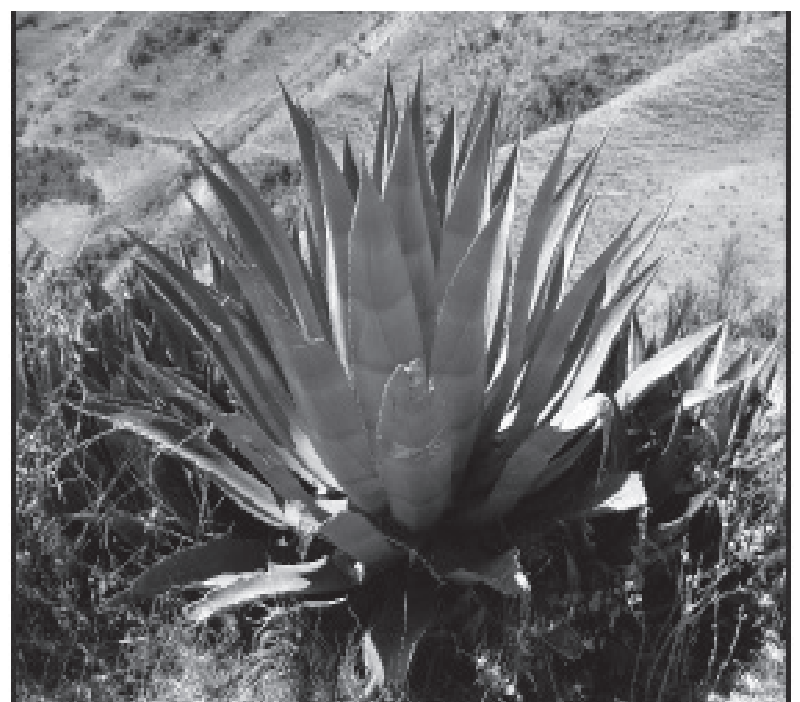

\section{PRUEBAS EXPERIMENTALES}

Para el presente estudio de investigación se han identificado plantas de cabuya azul de Huancavelica, a las cuales se le ha practicado una incisión en la base, por la cual fluye un líquido ligeramente viscoso que es recolectado para el análisis, el cual constituye el jugo o agua miel de cabuya.
Figura 2. Recolección del jugo de cabuya azul

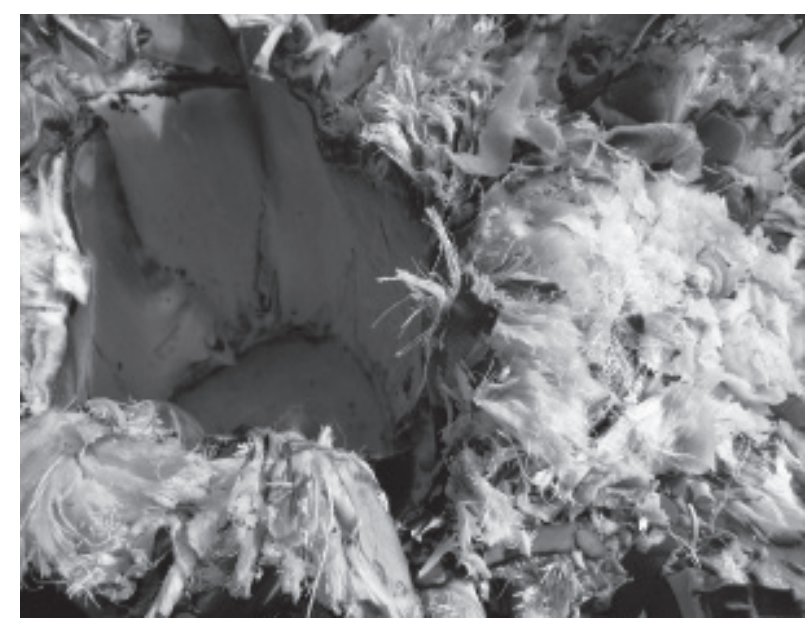

\section{MÉTODOS Y TÉCNICAS DE IDENTIFICACIÓN UTILIZADAS (1)}

Las técnicas de análisis utilizadas para determinar las propiedades fisicoquímicas del jugo de cabuya azul fueron:

- Refractómetro de 505 nm de longitud de onda, para la inulina.

- Refractómetro para los grados Brix del jugo.

- Picnómetro para la densidad.

- Viscosímetro de Ostwald para la viscosidad.

- Gravimetría para la fructosa.

\section{RESULTADOS EXPERIMENTALES}

Los resultados de los análisis de las propiedades fisicoquímicas de muestras de jugo o agua miel de cabuya azul confirman la presencia de inulina. Este resultado constituye la base para proponer su transformación industrial, a favor de la población de las zonas rurales identificadas.

En el cuadro siguiente se puede apreciar el contenido de inulina en la muestra patrón y en el jugo de cabuya.

Cuadro 1. Contenido de inulina en muestra patrón y jugo.

\begin{tabular}{|c|c|}
\hline Muestra & $\begin{array}{c}\text { Contenido de Inulina } \\
\text { (g/100 g de muestra) }\end{array}$ \\
\hline Inulina patrón & 74.82 \\
Jugo de cabuya & 33.9 \\
\hline
\end{tabular}

Fuente: FAMALAB 
El análisis fisicoquímico de la muestra de jugo o agua miel de cabuya obtenida, se aprecia en el cuadro N 2.

\section{INTERPRETACIÓN Y DISCUSIÓN DE RESUL- TADOS}

Los resultados obtenidos, que son el promedio de tres muestras analizadas, demuestra que la cabuya azul tiene alto contenido de inulina con un nivel de $33.9 \%, 43.05 \mathrm{~g}$ de inulina por $100 \mathrm{~mL}$ de jugo.

El resultado de los grados Brix (14), mostrados en el cuadro 2, reflejan una cantidad apreciable de azucares o de sólidos totales.

Además, se determinaron la densidad y la viscosidad de la muestra, cuyo resultado que figura en el cuadro 2, es un indicador de un alto contenido de sólidos totales.

\section{CONCLUSIONES}

La cabuya azul peruana tiene una alto contenido de inulina, $33.9 \%$.

El contenido de sólidos totales que se sitúa en $14^{\circ}$ Brix, es un indicador de un alto contenido de edulcorantes.
Los resultados de los análisis fisicoquímicos realizados, muestran altos niveles de productos alimenticios y medicinales del jugo de cabuya azul.

\section{RECOMENDACIONES}

En base a los resultados obtenidos se recomienda continuar estudios de investigación aplicada, para obtener miel y panela a partir del jugo de cabuya, en coordinación con las comunidades de las regiones donde existe este recurso natural, que constituirían una posibilidad de mejorar la calidad de vida de la población involucrada.

\section{REFERENCIAS BIBLIOGRÁFICAS}

[1] Carla Vargas Vázquez (2009). Obtención de insumos de interés industrial a Partir de las Fructanas del Agave Mescalero Potosino, Tesis para obtener el grado de Maestro en Ciencias Agrícolas, Michoacán, México, 2009.

[2] R. c. Gómez - Ayala y otros. El Agave Americano como una nueva Fuente de inulina y Oligofructosa., UNAM, México.

Cuadro 2. Propiedades fisicoquímicas del jugo de cabuya azul

\begin{tabular}{|c|c|c|c|c|}
\hline \multicolumn{5}{|c|}{ PROPIEDADES } \\
\hline $\begin{array}{c}\text { Contenido de } \\
\text { inulina }\end{array}$ & $\begin{array}{c}\text { Contenido de } \\
\text { fructosa }\end{array}$ & Grados Brix & Densidad & Viscosidad \\
\hline $56.60 \mathrm{~g} / 100 \mathrm{~g}$ jugo & $5.60 \mathrm{~g} / 100 \mathrm{~g}$ jugo & $14 \mathrm{~g} / 100 \mathrm{~g} \mathrm{jugo}$ & $1.27 \mathrm{~g} / \mathrm{mL}$ & $1.56 \mathrm{cp}$ \\
\hline
\end{tabular}

Fuente: FAMALAB. Elaboración propia. 